\title{
Small Vibration of System Including Rolling Friction with Starting Rolling Displacement"
}

\author{
Tadayoshi KOIZUMI $^{* *}$ and Katsuhira TAUE ${ }^{* * *}$ \\ **Department of mechanical engineering informatics, Meiji University \\ 1-1-1, HIgashimita, Tama-ku, Kawasaki, 214-8571, Japan \\ E-mail: tkoizumi@isc.meiji.ac.jp \\ ${ }^{* \star *}$ Graduate School of Science and Technology, Meiji University
}

\begin{abstract}
In this study, we present a curve of the starting rolling displacement, which considers the starting region in which the rolling friction force depends on the displacement. We then analyze the nonlinear vibration of a one degree-of-freedom system with rolling friction force depending on the displacement. From the analytical results, it was found that a stopping motion is recognized below the resonance point, and above the resonance point, the vibration wave shows a clear sinusoidal. The parameter $m$ that shows the energy loss in a system using a curve of the starting rolling displacement greatly affects the vibration characteristics, and the different characteristics appear in the case of $m \geqq 2$ and $m<2$.
\end{abstract}

Key words: Numerical Analysis, Forced Vibration, Nonlinear Vibration, Rolling Friction, Stopping Motion, Starting Rolling Displacement

\section{Introduction}

Basic studies are being promoted to clarify the mechanisms causing rolling friction. So far, adhesion, slippage, and elastic hysteresis loss have been investigated ${ }^{(1)}$. The previous studies have also clarified a very small area where the friction force increases like the exponential function with the rolling distance at the start of rolling ${ }^{(1)-(3)}$. Since rolling friction is much smaller than sliding friction in fields of engineering, the rolling contact mechanism is widely used for the sliding parts of machines requiring low friction. Also, the rolling-accompanied system behaviors are becoming very important in abrasion, rolling fatigue problems and precise decision.

For vibration analyses considering friction, the friction force is commonly used as constant value. However, as the starting displacement becomes as small as tens of microns, it becomes difficult to consider the friction force as a constant because there is a region dependent on the displacement. In this study, therefore, displacement-dependent friction force is expressed with a starting rolling displacement curve $^{(3)}$ by considering the starting rolling displacement region where the friction force is dependent on the displacement. Since this expression becomes a nonlinear equation, a numerical analysis such as the Runge-Kutta method is necessary. Then, the 4th-degree Runge-Kutta method is used, the vibration waveform, FFT analysis and phase plane and so on are investigated to clarify the vibration characteristics of a system with a starting rolling displacement.

\section{Starting Rolling Displacement Curve}

To express the displacement-dependent friction force in the region of the starting rolling displacement, the following starting rolling displacement curve, which is originally presented, is used ${ }^{(3)}$.

where $\xi=x / x_{c}$ and $g(\xi)=f(x) / f . f(x)$ is the rolling friction force dependent on the displacement, $f_{c}$ is the constant friction force when the displacement has increased, 


$$
\begin{aligned}
g(\xi) & =\xi(1-\log (\xi)) & & (m=2) \\
& =\frac{1}{2-m}\left(\xi^{m-1}-(m-1) \xi\right) & & (m \neq 2)
\end{aligned}
$$

$x$ is the rolling distance, $x_{c}$ is the width of the region of the starting rolling displacement.

Fig. 1 shows the calculated examples of the starting rolling displacement curve expressed by Eq.(1). As we can see from Fig. 1, the parameter $m$ characterizes the size of the hysteresis loop area formed within the starting rolling displacement area, and $m$ also represents the intensity of the energy loss. As the $m$ value becomes smaller, the hysteresis loop area increases. It was confirmed experimentally that the rolling friction force could be basically expressed by a curve of $m=2^{(2)}$. When $m=$ 1 , the friction force is constant and does not depend on the displacement.

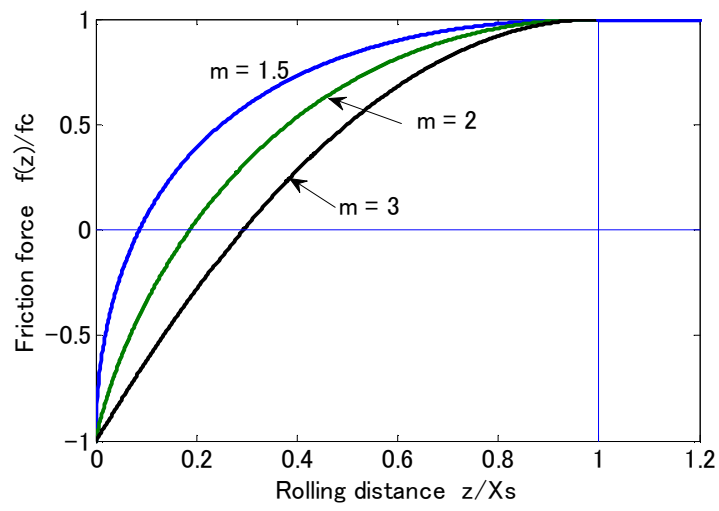

Fig.1 Starting Rolling Displacement curve

\section{Equation of Motion}

The vibration model is a 1-DOF system where mass $M$ is coupled with the base through a linear spring of the spring constant $k$. Mass $M$ is affected not only by the spring force (restoring force) but also by the external exciting force $P \sin (\omega t)$ and the rolling friction force $F(z)$. The free damped vibration with the rolling friction force $F(z)$ was analyzed by considering the starting rolling displacement, as already reported ${ }^{(4)}$.The equation of motion of this system can be expressed as

$$
M \ddot{x}+k x=P \sin (\omega t) \mp\left(2 f(z)-f_{c}\right)
$$

$F(z)$, representing the friction force, can be expressed as

$$
F(z)=2 f(z)-f_{c}
$$

Where $z$, introduced to calculate the displacement-dependent friction force, represents the rolling distance from the origin, where the direction of the movement of the mass is inverted. The sign of the friction force is $-F(z)$ when the mass moves in the positive direction of the $x$ axis and $+F(z)$ when it moves in the negative direction. Eq. (2), where $\omega_{n}{ }^{2}=k / M, X_{s}=P / k$, and $\alpha=f_{c} / P$, can be made dimensionless with $X=x / X_{s}$ and $Z=z / X_{s}$ as

$$
\begin{aligned}
& \ddot{X}=-\omega_{n}{ }^{2} X+\omega_{n}{ }^{2} \sin (\omega t) \mp F(Z) \\
& F(Z)=\omega_{n}{ }^{2} \alpha(2 g(Z)-1)
\end{aligned}
$$

The analysis of the nonlinear vibration equation and that of the system with a friction have been introduced so $\mathrm{far}^{(5)(6)(7)}$. The study on the steady-state response of a system with a micro slip friction is also made ${ }^{(8)}$. In this study, the 4th-degree Runge-Kutta method is used for analysis with Eq. (4). For analysis of a friction system, the time step needs to be short. Here, the time step for the stationary state is 
set to $1 / 6000$ of the frequency of excitation in case of $\omega / \omega_{n}<1$, and $1 / 12000$ of the frequency of excitation in case of $\omega / \omega_{n} \geqq 1$.

\section{Results of Numerical Analysis}

This section gives the results of the numerical analysis. For numerical calculation, the initial displacement $X_{0}$ is 0 and the initial velocity $d X_{0} / d t$ is 0 . It is known that a system is affected by friction force, and it shows a stopping motion in the region where the resonance point is below the vibration frequency ratio ${ }^{(9)(10)(11)(12)}$. And the stopping motion in the nonlinear vibration such as Duffing's equation ${ }^{(13)}$ is also observed.

Fig. 2 shows the vibration waveform, the friction force, the phase plane and the FFT analytical result when the vibration frequency ratio $\Omega=\omega / \omega_{n}$ is 0.3 below the resonance point and 1.5 above the resonance point. The curvature parameter $m$ is 2 and the ratio $\alpha$ of the friction force to the external force is 0.5 .

As we can see from the results of this analysis, when the vibration frequency ratio $\Omega=\omega / \omega_{n}$ in Fig. 2(a) is 0.3 below the resonance point, the flat portion of waveform has occurred and the waveform don't show smooth sinusoidal curve.

As shown from the phase plane of $\Omega=0.3$, we can observe the vibration behaviors that the velocity becomes almost constant or close to 0 . This indicates that a stopping motion is produced during the vibration. From the analytical results of $\Omega=1.5$ above the resonance point, it can be known that the vibration waveform becomes clearly sinusoidal and the phase plane becomes elliptic. In the FFT analysis, the frequency of an odd multiple of the externally excited frequency $\Omega=0.3$ and $\Omega=1.5$ appeared. It is well known that the Fourier series for constant friction force is represented by only the odd terms. When the one period is also constituted by two straight line, $(\mathrm{f}(\mathrm{x})=\mathrm{x}: 0<\mathrm{t}<1, \mathrm{f}(\mathrm{x})=-\mathrm{x}+1: 1<\mathrm{t}<2)$, the Fourier series of this function is represented by the odd terms. The terms of odd frequency might appear in system of the rolling friction force depending on the displacement. This is a characteristic of nonlinear vibration.
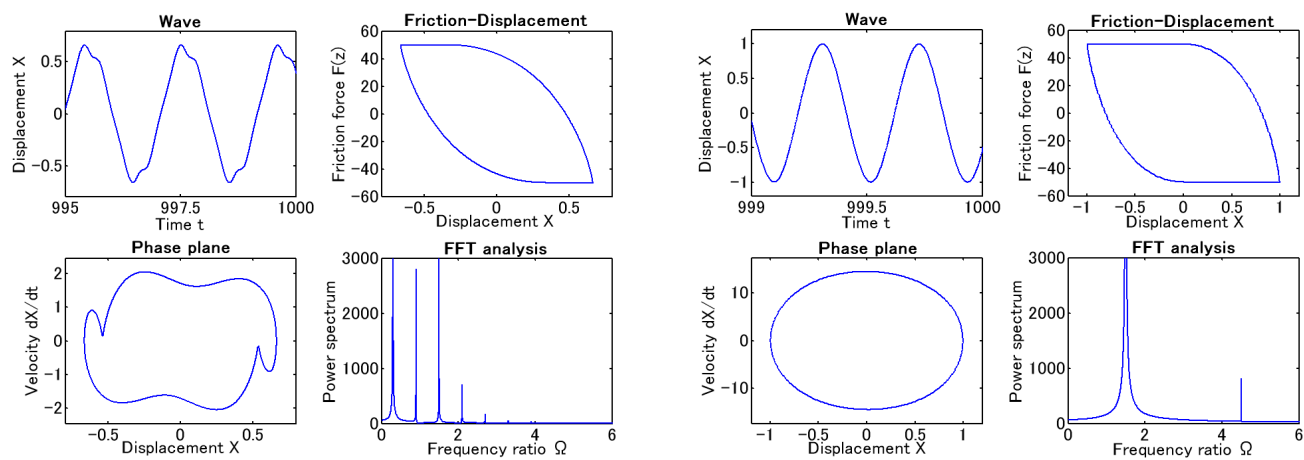

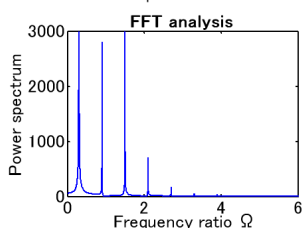

(a) $\Omega=0.3$
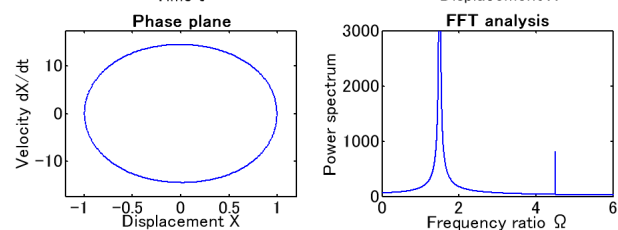

(b) $\Omega=1.5$

Fig.2 Vibration characteristics $\quad(m=2, \quad \alpha=0.5)$

In Fig. 3 and 4 to make clear the vibration characteristics, the change of acceleration, velocity, spring force and friction force are shown between 1 cycle. Simultaneously, the vibration waveform, the phase plane and FFT analysis are together shown in Fig.3. Here, the parameter $m$, representing the intensity of the energy loss, is set to 1.2 and $\alpha(=f c / P)$ is set to 0.9 . Fig. 3 shows the case of vibration frequency ratio $\Omega=0.3$ as an example of obvious stopping motion. In this case, the friction force $F(z)$ changes in the stairs state with time at the inverse position and becomes smoothly with the passage of time. In the case of vibration 

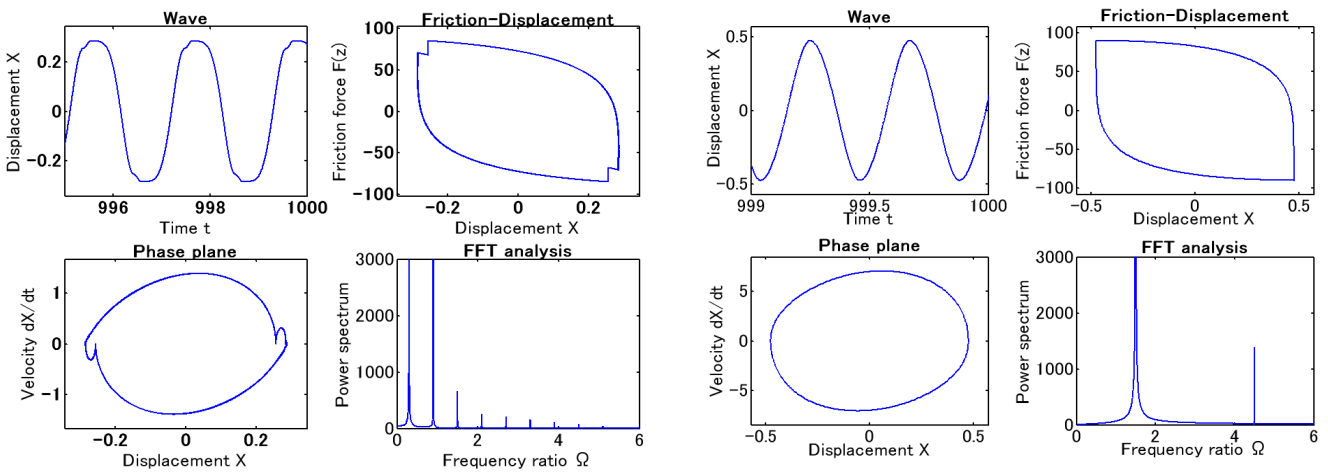

Fig.3(a) Vibration characteristics $(\Omega=0.3)$

Fig.4(a) Vibration characteristics $(\Omega=1.5)$
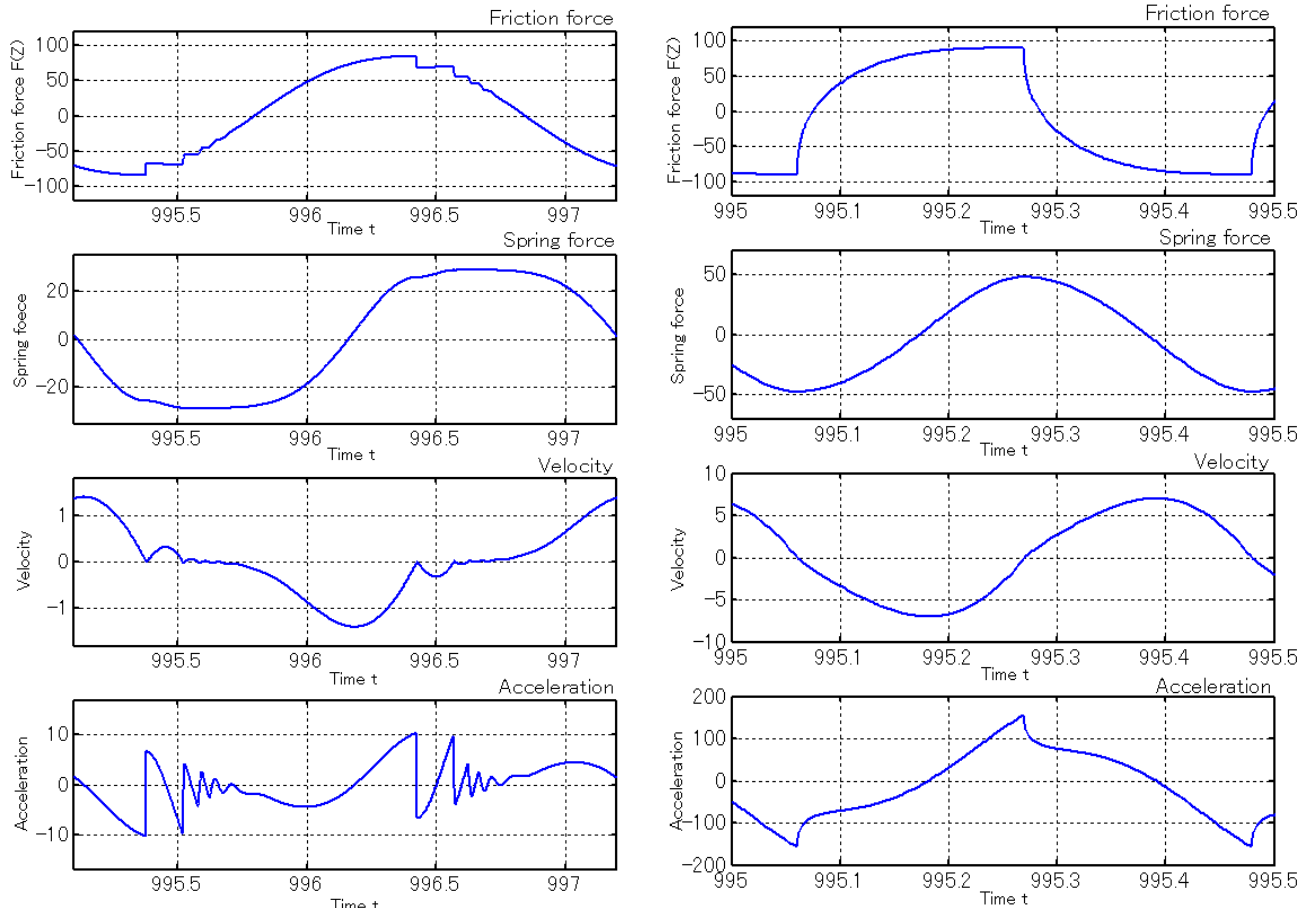

Fig.3(b) Change of Friction force, Velocity and acceleration $(\Omega=0.3$,

Fig.4(b) Vibration characteristics $(\Omega=1.5)$

frequency ratio $\Omega=0.3$, we can also see stopping motion in the region where the acceleration is producing damped vibration. The velocity is also producing a vibration, while it is accompanied by the stopping motion at the inverse position.

Fig. 4 shows the case of $\Omega=1.5$ that the vibration wave is sinusoidal. In this case

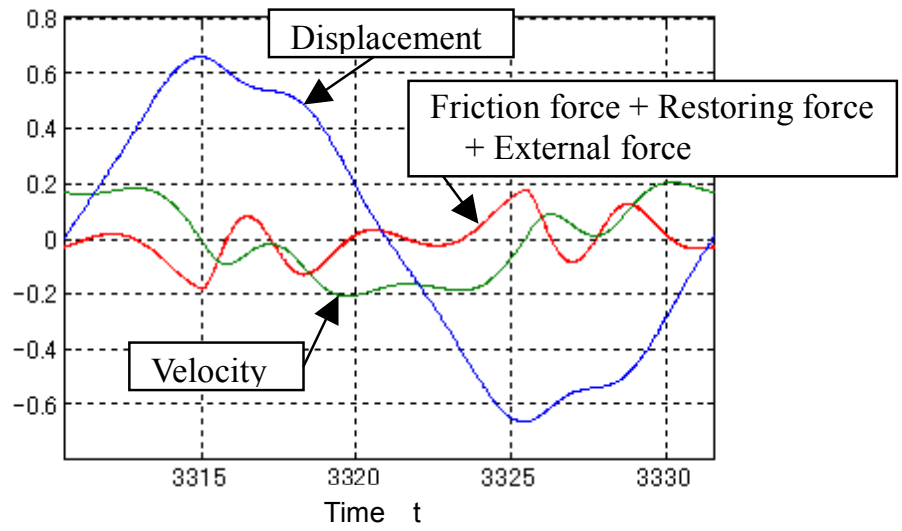

Fig.5 Change of displacement, velocity and sum of three force $(\mathrm{m}=2, \quad \Omega=0.3, \quad \alpha=0.5)$ 
the change of velocity becomes smoothly, the acceleration does not show a damped vibration when the direction of movement is inverted. This is because the acceleration is greater than the friction. Thus, in a region of stopping motion, the change of acceleration exceeds 0 and the acceleration shows the damped vibration at the inverse position as shown in Fig.3(b).

Change of the displacement, velocity and sum of three force (friction force, spring force, external force) are shown in Fig.5. As shown in figure, there are two cases in which the three force becomes 0 .. One is the case that the velocity becomes 0 and another is the case that the velocity gradient (the acceleration) becomes 0 . When the velocity becomes 0 , the movement of displacement is observed as if the displacement does not change with the passage of time.

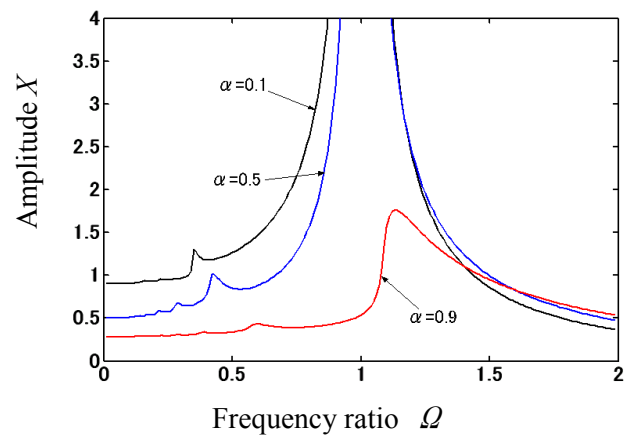

(a) $m=2.0$

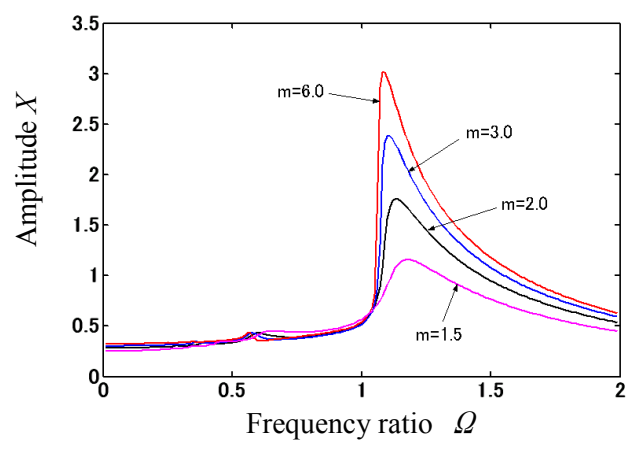

(b) $\alpha=0.9$

Fig.6 Resonance curve

The resonance curve is shown in Fig. 6. Fig. 6(a) shows the resonance curve after setting the energy loss parameter $m$ to 2.0 and varying the ratio $\alpha$ of the friction force to the external force as the parameter. As the value of $\alpha$, representing friction force, becomes greater, the value of the amplitude is smaller, and the resonance curve shows disturbance in the region where the vibration frequency ratio, representing stopping motion, is small. The clear occurrence of disturbance, the displacement amplitude increases like as a projection, are near $\Omega=0.5$. In the region where the vibration frequency ratio exceeds the resonance point, the displacement amplitude varies very smoothly, the resonance curve is not disturbed and the displacement waveform is close enough to be a sinusoidal wave, as the example of $\Omega=1.5$ is already shown in Fig.4. The resonance point shifts to larger frequency as the $\alpha$ value increases.

Fig. 6(b), in which the influence of parameter $m$ is remarkable, shows the resonance curve drawn by setting $\alpha$ to 0.9 and varying the $m$ value as the

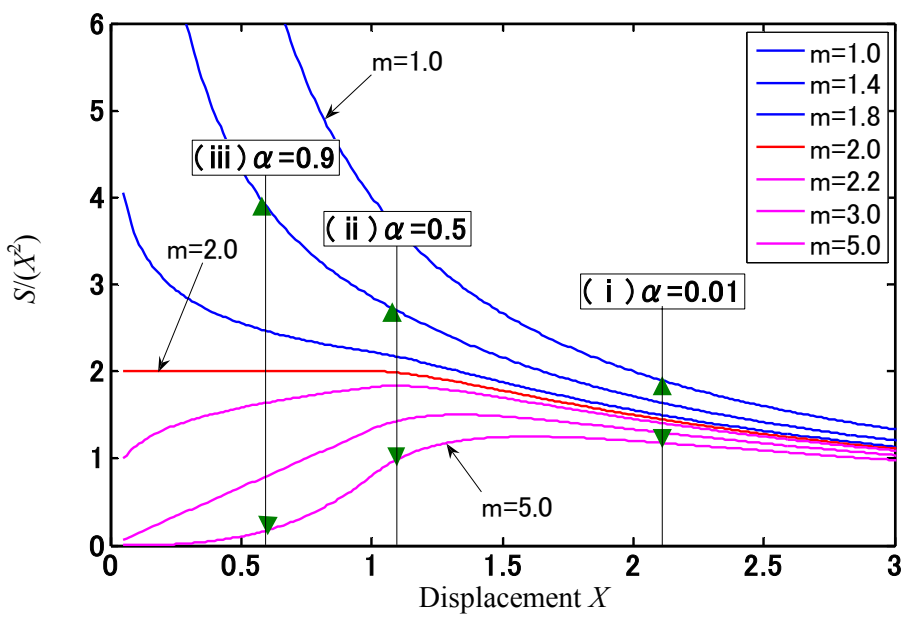

Fig.7 Loss energy of system 
parameter. Since the energy loss diminishes as the $m$ value increases, the amplitude at the resonance point becomes large. The resonance point shifts to larger frequency as the $m$ value increases. Though the resonance curve is disturbed at near $\Omega=0.5$, the disturbance is small because of the large value of $\alpha=0.9$, which indicates the friction force.
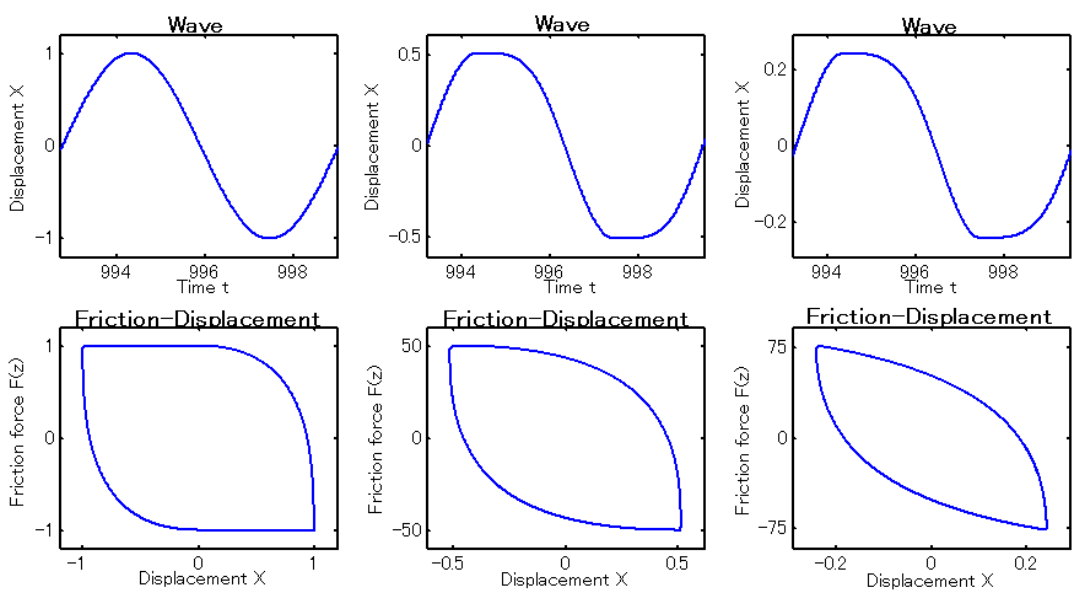

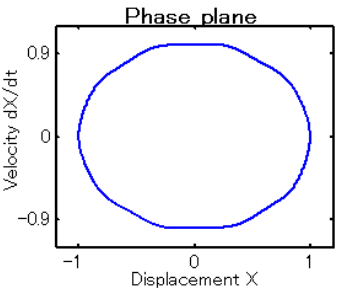

(i) $\alpha=0.01$
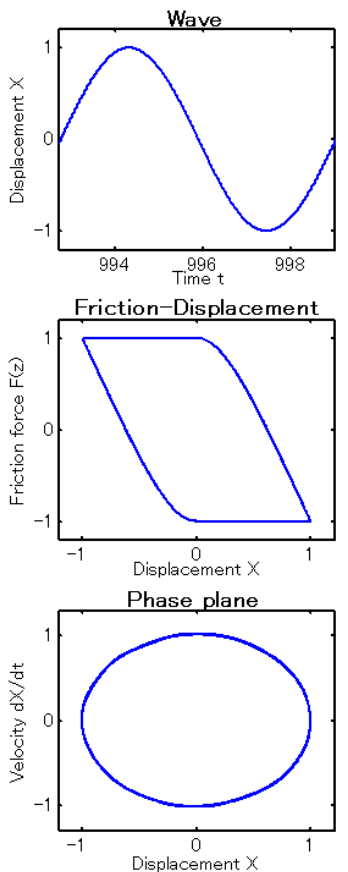

(i) $\alpha=0.01$

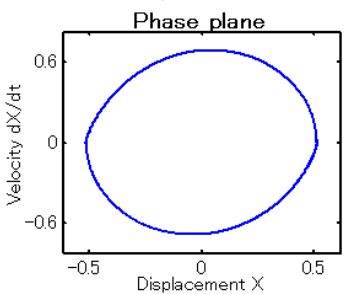

(ii) $\alpha=0.5$

(a) $m=1.4$
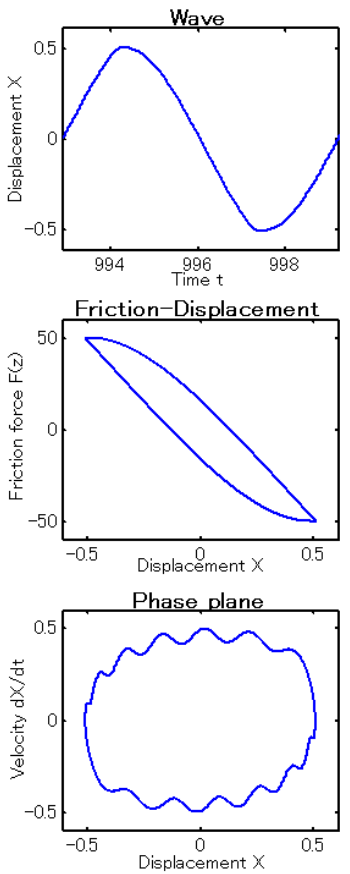

(ii) $\alpha=0.5$

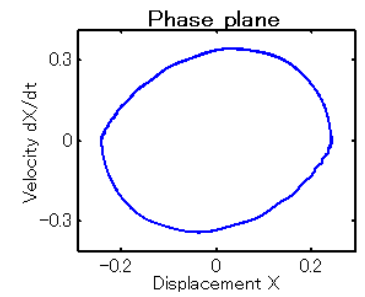

(iii) $\alpha=0.9$
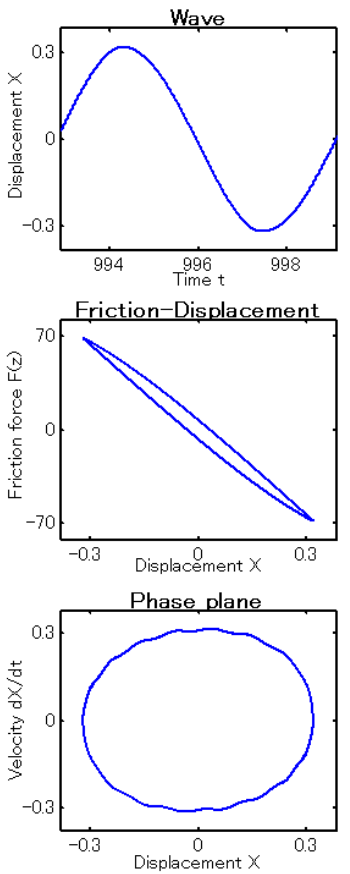

(iii) $\alpha=0.9$

(b) $m=5$

Fig.8 Vibration characteristics

（ $\Omega=0.1 ）$ 
Fig. 7, which is the result of studying the loop area of the friction force, shows the relationship between energy loss and amplitude. The vertical axis indicates the ratio of the energy loss , $S$, to the elastic energy of the spring, $X^{2}$, that the loop area $S$ is divided by the square of the amplitude. The horizontal axis indicates the
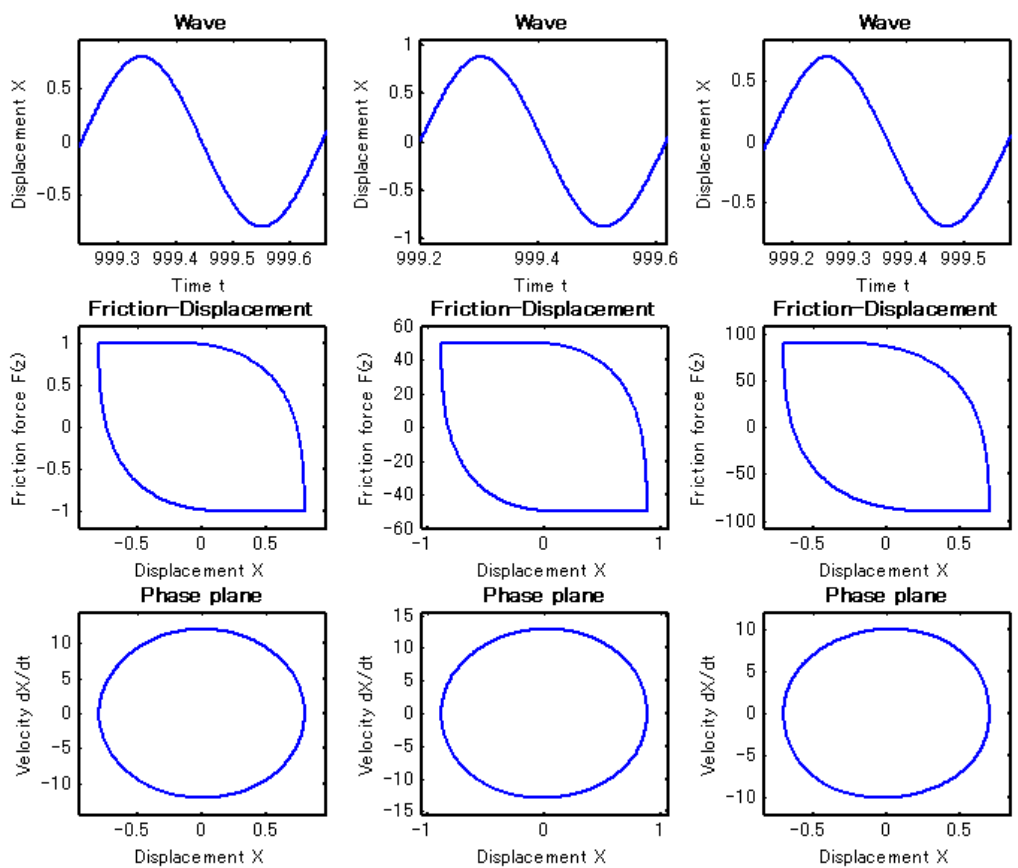

(i) $\alpha=0.01$

(ii) $\alpha=0.5$

(iii) $\alpha=0.9$

(a) $m=1.4, \Omega=1.5$
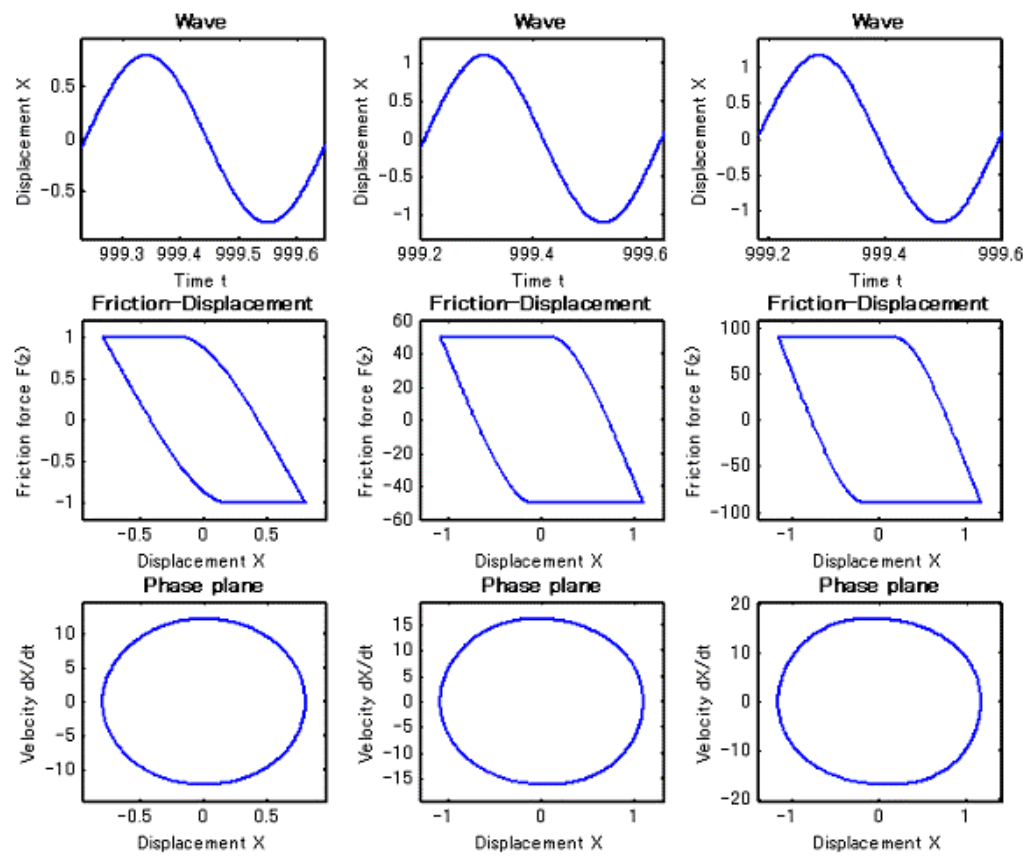

(i) $\alpha=0.01$

(ii) $\alpha=0.5$

(iii) $\alpha=0.9$

(b) $m=5, \Omega=1.5$

Fig.9 Vibration characteristics 
amplitude where $0 \leq X \leq 1$ is the starting rolling displacement region. $S / X^{2}$ implies a loss factor. As shown from Fig.7, we see that the loss factor increases as the amplitude diminishes when $m<2$, but the loss factor shows a maximum value during vibration about the starting rolling displacement when $m>2$. The loss factor changes as the standard for $m=2$.

In Fig.8 and Fig.9, the vibration characteristics about the starting rolling displacement have been investigated. Fig.8(a),(b) show the displacement wave, the friction force and the phase plane. The value of $\Omega$ is 0.1 . In order to compare the effect of the system behaviors when $m<2$ and $m>2$, the result of $m=1.4$ is shown in Fig.8(a) and $m=5$ is shown in Fig.8(b). From left side of Figure, the $\alpha$ value is $0.01,0.5$, and 0.9 , and the positions of amplitude are described in symbol $\triangle, \nabla$, as already shown in Fig.7.

In case of $\Omega=0.1, m=1.4$ when the $\alpha$ value increases, the value of displacement amplitude decreases, and the area of hysteresis loop also decreases. The form of the ellipse in phase plane is distorted with increasing the $\alpha$ value. Though the same characteristics are also producing in case of $m=5$, the contour of ellipse drawn in phase plane undulates at $\alpha=0.5$, and the stopping motion occurs obviously, where the vibration is at the starting rolling displacement of $-0.5 \leq X \leq 0.5$, as shown in Fig.8(b)( ii ). When $m>2$, the loss factor shows maximal during vibration at the starting rolling displacement. In case of $\Omega=0.1$ below the resonance point, when the value of $\alpha$ becomes large, a stopping motion remarkably arises.

The vibration characteristics in case of $\Omega=1.5$, where the vibration frequency ratio exceeds the resonance point, are shown in Fig.9. In this region the waveform and the phase plane varies smoothly. As discussed above, if the friction force is expressed by a starting rolling displacement curve, the system vibration characteristics tend to change before and after $m=2$. The parameter $m$ that shows the energy loss in a system using a curve of the starting rolling displacement greatly affects the vibration characteristics, and the different characteristics appear in the case of $m \geqq 2$ and $m<2$. The stopping motion occurs in the region where the vibration frequency ratio is below the resonance point, but not where the ratio is above the resonance point where the waveform becomes clearly sinusoidal.

\section{Conclusion}

In this study, a starting rolling displacement curve was introduced to express displacement dependent friction force, and the vibration under the effect of rolling friction accompanied by starting rolling displacement was numerically analyzed to study the vibration characteristics. This clarified that stopping motion occurs in the region where the vibration frequency ratio is below the resonance point, but not where the ratio is above the resonance point where the waveform becomes_clearly sinusoidal. In a region of stopping motion, the change of acceleration exceeds 0 and the acceleration shows a damped vibration at the inverse position. The sum of friction force, spring force, external force become 0 , and the velocity also becomes 0 . This study clarified that the system vibration characteristics change, depending on whether the parameter $m$ contained in the starting roll displacement curve is smaller or greater than 2 .

\section{References}

(1) BOWDEN, F.P. and TABOR, D.,THE FRICTION AND LUBRICATION OF SOLIDS,PART II,(1964), p.277, OXFORD,

(2) T.KOIZUMI, H.SHIBAZAKI, T.NISHIO, N.NISHIWAKI, Study on Rolling Friction Behaviour of Small Displacement of Starting Rolling Friction - J.of JSLE., Vol.27,No.9 (1982), pp.690-696 (in Japanese)

(3) H.SHIBAZAKI, T.KOIZUMI, Study of a Curve of the Small Displacement of Starting Rolling Friction, J. of JSLE, Vol.29, No.1 (1984), pp.50-55 (inJapanese)

(4) T.KOIZUMI, O. KURODA, Analysis of Damped Vibration of a System with Rolling 
Friction - Rolling Friction Depends on the Displacement -, Japanese Journal of Tribology, Vol.35, No.6 (1990), pp.435-439

(5) A.GURAN, F.PFEIFFER \& K.POPP, Dynamics with Friction, Part I,(2001), p.1, World Science

(6) SHAW, S.W., On the Dynamic Response of a System with Dry Friction, Journal of Sound and Vibration, Vol.108,No.2 (1986), pp.305-325

(7) B. F. FEENY and J. W. LIANG, A DECREMENT METHOD FOR THE SIMULTANEOUS ESTIMATION OF COULOMB AND VISCOUS FRICTION, Journal of Sound and Vibration, Vol.195,No.1 (1996), pp.149-154

(8) C.H. MENO, J. BIELAK, J.K. GRIFFIN, THE INFLUENCE OF MICROSLIP ON VIBRATORY RESONANSE, PART 1: A NEW MICROSLIP MODEL, Journal of Sound and Vibration, Vol.,107,No.2 (1986), pp.279-293

(9) C.PIERRE, A.A. FERRI, E.H. DOWELL, Multi-Harmonic Analysis of Dry Friction Damped Systems Using an Incremental Harmonic Balance Method, ASME J. Appl. Mech Vol.52,No.4 (1985), pp.958-964

(10) E. MARUI, S. KATO, Forced Vibration of a Base-Excited Single-Degree-of-Freedom System With Coulomb Friction, ASME, J. Dynamic System, Measurement and Control Vol.106,No.4 (1984), pp.280-285

(11) A.AKAY, Acoustics of friction, J. Acoust. Soc. Am., Vol.111, No.4 (2004), p.1532

(12) H. K. HONG, C.S. LIU, COULOMB FRICTION OSCILLATOR: MODELLING AND RESONANSES TO HARMONIC LOADS AND BASE EXCITATIONS, Journal of Sound and Vibration, Vol.229, No.5 (2000), pp.1171-1192

(13) F.C. MOON, Chaotic Vibration, (2004), p.77, WILEY 